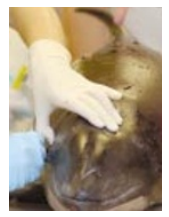

Noise annoys

Moves to relax sonar rules spark marine protest p549

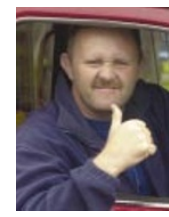

Cabbing fever

Ig Nobel prizes give study of taxi-drivers the thumbs up p550

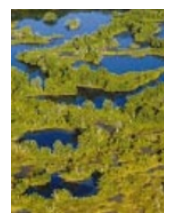
Judgement day

Everglades project flounders after judge is ousted p551

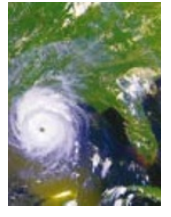
Weather report Japan throws the switch for high-res climate model p552

\title{
Magnetic pioneers net Nobel for putting medicine in the picture
}

Helen Pearson, London

For allowing doctors to peer into previously impenetrable parts of the human body, two physicists have scooped the 2003 Nobel Prize in Physiology or Medicine. Paul Lauterbur of the University of Illinois in Urbana and Peter Mansfield of the University of Nottingham, $\mathrm{UK}$, share the prize for their contribution to the development of magnetic resonance imaging (MRI).

Before the advent of MRI in the 1970s, doctors struggled to examine organs and soft tissues in people using X-rays. Today, MRI clinicians and medical researchers alike can readily view details of the internal structure of patients' entire bodies.

Lauterbur and Mansfield kick-started this revolution in medical imaging by tweaking a technique called nuclear magnetic resonance (NMR), primarily used for investigating molecular structures. When placed in a magnetic field, the nuclei of certain atoms, including hydrogen, line up. Subjected to a blast of radio waves, they absorb this radiation and emit it again as an NMR signal from which the structure of the molecules in which the atoms sit can be deciphered.

While working at the State University of New York at Stony Brook, Lauterbur came up with the idea of using a graded magnetic field that increased in strength from one side of an object to the other to create a two-dimensional image of the object's internal structure. This is based on the idea that nuclei absorb and emit radio waves at different frequencies, depending on the strength of the magnetic field in which they are held.

In a seminal paper, Lauterbur produced crude images of two glass capillaries filled with water (P. C. Lauterbur Nature 242, 190-191; 1973). His manuscript was initially rejected by Nature, but his protests proved successful. "I congratulate you on eventually giving in to my anguish," he told Nature this week, hours after the prize was announced.

From the beginning, Lauterbur believed that the technique could be used to image live animals, rather than killing them to remove and study their tissues. "I thought there must be another way," he says.

But at the time, it was difficult to envisage the technique evolving to give us the sophisticated medical images that are now routine - nearly 60 million of them were taken in 2002. "No one could imagine doing this to people," explains radiologist Paul Bottomley of Johns Hopkins University in Baltimore, Maryland.

It was Mansfield who, in a series of papers, provided imaginative leaps to turn MRI into a useful clinical tool. "His talent is tenacity and coming up with good ideas," says Bottomley.

The first advance made by Mansfield was to show that it is possible to selectively image a particular two-dimensional slice of an object held in a graded magnetic field by delivering the blast of radio waves at a particular frequency. Second, he developed techniques to manipulate the applied magnetic fields and algorithms to interpret the resulting cacophony of radio signals that enabled images to be pieced together in just a matter of seconds.

Mansfield's work has allowed researchers and clinicians to take snapshots of fastmoving events, such as the beating of the heart. It has also been vital in the development of brain scanning by functional MRI, in which mental activity can be tracked by monitoring changes in blood flow. "It's been critical," says Paul Matthews, director of the

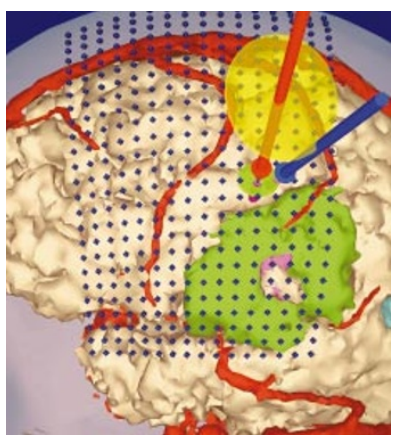

MRI in action: a brain tumour (green) is marked for surgery.
Centre for Functional MRI of the Brain at the University of Oxford, UK.

A Nobel for MRI has been long anticipated but, because its development has involved many steps, deciding who to reward won't have been easy. "It's been a hot potato for the Nobel prize committee," suggests John Griffiths of St George's Hospital Medical School, London, who uses MRI to diagnose and study cancer - one of its most important clinical applications.

In the end, the Nobel committee seems to have awarded the prize specifically for the initial developments involving the use of graded magnetic fields. But experts say that this does not preclude recognition of other contributors to MRI in the future. "It leaves the way open," says Stephen Keevil, a magnetic resonance physicist at Guy's Hospital, London.

www.nobel.se 